Comment. Math. Helv. 73 (1998) 584-602

(C) 1998 Birkhäuser Verlag, Basel

0010-2571/98/040584-19\$1.50+0.20/0

Commentarii Mathematici Helvetici

\title{
Stable constant mean curvature hypersurfaces in some Riemannian manifolds
}

Sebastián Montiel*

\begin{abstract}
We determine all stable constant mean curvature hypersurfaces in a wide class of complete Riemannian manifolds having a foliation whose leaves are umbilical hypersurfaces. Among the consequences of this analysis we obtain all the stable constant mean curvature hypersurfaces in many nonsimply connected hyperbolic space forms.
\end{abstract}

Mathematics Subject Classification (1991). Primary 53A10; Secondary 49Q20.

Keywords. Constant mean curvature, hypersurface, stability.

\section{Introduction}

Compact hypersurfaces $\Sigma^{n}$ with constant mean curvature in a Riemannian manifold $M^{n+1}$ are first order solutions to an isoperimetric problem: they are critical points of the area functional, admissible variations being only those that leave a certain enclosed volume function fixed. In this setting, it is natural to study constant mean curvature compact hypersurfaces which are stable with respect to that variational problem, i.e., those for which the index form $Q$ arising from the second variation of the area is nonnegative for all admissible variations. More precisely (see $[\mathrm{BdC}, \mathrm{BdCE}])$, those satisfying

$$
Q(h)=\int_{\Sigma}\left\{|\nabla h|^{2}-\left(S(N, N)+|\sigma|^{2}\right) h^{2}\right\} d A \geq 0,
$$

for any $h \in C^{\infty}(\Sigma)$ such that $\int_{\Sigma} h d A=0$. There $\nabla h$ stands for the gradient of $h$, $N$ is a unit normal vector field on $\Sigma$ (we will assume $\Sigma$ to be orientable and $M$ to be oriented), $S$ is the Ricci tensor of the ambient manifold $M$ and $\sigma$ the second fundamental form of the immersion.

The classification of stable compact hypersurfaces with constant mean curvature was acomplished by Barbosa and do Carmo $([\mathrm{BdC}])$ when the ambient space

${ }^{*}$ Research partially supported by a DGICYT grant PB94-0796. 
$M^{n+1}$ is the Euclidean space $\mathbb{R}^{n+1}$ and continued by themselves and Eschenburg $([\mathrm{BdCE}])$, and independently by El Soufi and Ilias in $[\mathrm{EI}]$ and by Heintze in $[\mathrm{H}]$, when $M^{n+1}$ is either the sphere $\mathbb{S}^{n+1}$ or the hyperbolic space $\mathbb{H}^{n+1}$. Their results can be jointly stated as follows:

The only stable compact orientable hypersurfaces with constant mean curvature immersed in a simply connected space form are the umbilical (round) spheres.

Besides this case where the ambient space is a simply connected space form, this kind of hypersurfaces has been completely classified only when $n=2$ and $M^{n+1}$ is the three dimensional real projective space $\mathbb{R} P^{3}$, by Ritoré and Ros [RR1]. In this nonsimply connected low dimensional case, one can observe that an easy but nontrivial topology in the ambient space yields the apparition of different types of orientable compact stable constant mean curvature surfaces requiring more sophisticated classification technics. Increasing topology or changing compactness by completeness hardens considerably the problem (see [RR2] and references therein).

In this paper we will classify all orientable compact stable constant mean curvature hypersurfaces in many nonsimply connected hyperbolic space forms. More precisely, particular cases of Corollaries 7 and 8 (see Remarks 3 and 4), will give

Let $\Gamma$ be a subgroup of $\mathrm{Iso}^{+}\left(\mathbb{H}^{n+1}\right)$ acting properly and discontinuously. Suppose that $\Gamma$ fixes a given horosphere $H_{0}$ (respectively, hyperbolic hyperplane). Then, the connected components of an orientable compact stable constant mean curvature hypersurface in the quotient $\mathbb{H}^{n+1} / \Gamma$ are either umbilical (round) spheres or $H / \Gamma \subset \mathbb{H}^{n+1} / \Gamma$, where $H$ is a horosphere (respectively hyperplane) parallel to $H_{0}$. Moreover, there is at most one connected component of the first type.

Notice that the subgroup of Iso ${ }^{+}\left(\mathbb{H}^{n+1}\right)$ consisting of the orientation preserving isometries fixing a given horosphere (respectively hyperplane) can be naturally identified with Iso $\left(\mathbb{R}^{n}\right)$ (respectively Iso $\left(\mathbb{H}^{n}\right)$ ). So, the result above can be applied to many hyperbolic space forms. It is also relevant to remark that, when the ambient space is a simply connected space form, stable hypersurfaces are necessarily connected. In our setting, examples will convince us that other is the case. Anyway, there are situations where we will be able to assert something more about the combination of the possible connected components. In fact, we will obtain in Remark 4 just after Corollary 8 that

Let $\Gamma$ be a subgroup of $\mathrm{Iso}^{+}\left(\mathbb{H}^{n+1}\right)$ acting properly and discontinuously and fixing a given horosphere $H_{0}$. Then, an orientable compact constant mean curvature hypersurface immersed in the quotient $\mathbb{H}^{n+1} / \Gamma$ is stable if and only if it is either an umbilical (round) sphere or a finite number of $H / \Gamma$, where $H$ means a horosphere parallel to $H_{0}$. 
The reason because we are able to classify stable constant mean curvature hypersurfaces in precisely these two types of space forms is that they can be described as certain warped products (see [BiOn,On]). In fact, we will achieve the classification of all orientable compact stable constant mean curvature hypersurfaces in a wider class of Riemannian manifolds having nonconstant curvature which can described in terms of those warped products. We will see in Corollary 6 that

If $f$ is a positive nonconstant solution to the Jacobi equation $f^{\prime \prime}+\kappa f=0$, $\kappa \in \mathbb{R}$, and $P^{n}$ is a Riemannian manifold whose Ricci curvature $S_{P}$ satisfies $S_{P} \geq(n-1)\left\{f^{2}+\kappa f^{2}\right\}$, then the connected components of any orientable compact stable constant mean curvature hypersurface into the warped product $\mathbb{R} \times_{f} P^{n}$ are of two types: either umbilical round spheres or slices $\{s\} \times P^{n}$, $s \in \mathbb{R}$. The first case occurs only when $P^{n}$ has constant curvature (in a certain region) and the second one only when $P^{n}$ is compact. Moreover, there is at most one connected component of the first type and only one connected component when $\kappa \geq 0$.

We must point out that when $\kappa<0$ the warped products $\mathbb{R} \times_{f} P^{n}$ provide us many Riemannian manifolds which are complete. The procedure that we will use for studying compact hypersurfaces in these ambient spaces is to use the existence on such warped products of a nontrivial closed conformal field to obtain certain integral formulae generalizing the classical Minkowski formulae. This has been already done in $[\mathrm{M}]$ to obtain analogues to the Liebmann ([J,Li] $)$ and Alexandrov ([A]) theorems on compact constant mean curvature hypersurfaces in Euclidean spaces.

\section{Closed conformal fields and induced foliations}

The ambient spaces $M^{n+1}$ that we are going to consider in order to study stability of compact constant mean curvature hypersurfaces can be described as certain warped products with a 1-dimensional factor (see Section 3 below). One of the relevant features of these Riemannian manifolds (which include the three simply connected space forms) is the existence of a nontrivial closed conformal field.

Thus, consider a Riemannian manifold $M^{n+1}$ endowed with a nontrivial closed conformal field $X$, that is, such that there exists a function $\phi \in C^{\infty}(M)$ with

$$
\nabla_{u} X=\phi u \quad \text { for every vector } u \in T M,
$$

where $\nabla$ stands for the Levi-Civita connection on $M$ associated to the Riemannian metric $\langle$,$\rangle . This geometrical situation has been widely considered in the literature,$ either directly imposed on a Riemannian manifold (see, for example, $[\mathrm{O}, \mathrm{TW}]$ and references therein), or related to other assumptions such as the existence of some function whose Hessian satisfies an Obata type condition [K,Ta]; the fact that $M$ 
is a Lagragian submanifold of $\mathbb{C}^{n}$ with conformal Maslov class [RU]; and, finally, the fact that $M$ is a warped product (in the sense of [BiOn] or [On], p. 204). It is also related with the existence of a certain class of foliations whose leaves are totally umbilical.

From definition (2), we immediately obtain

$$
\nabla|X|^{2}=2 \phi X=\frac{2}{n+1}(\operatorname{div} X) X .
$$

By taking covariant derivatives in (3), we may compute the Hessian of the function $|X|^{2}$. In fact we have

$$
\left(\nabla^{2}|X|^{2}\right)(u, v)=2(u \phi)\langle X, v\rangle+2 \phi^{2}\langle u, v\rangle,
$$

for every $u, v \in T M$. From this, because both the Hessian $\nabla^{2}|X|^{2}$ and the metric $\langle$,$\rangle are symmetric tensors, we obtain$

$$
(u \phi)\langle X, v\rangle=(v \phi)\langle X, u\rangle
$$

for all $u, v$ tangent to $M$. Hence

$$
|X|^{2} \nabla \phi=(X \phi) X \quad \text { that is }|X|^{2} \nabla \operatorname{div} X=(X \operatorname{div} X) X .
$$

Using these equalities and taking into account some results in $[\mathrm{O}]$, one can prove the following Proposition 1, where we will gather some well-known facts for Riemannian manifolds admitting such a vector field (see [M,RU] for a detailed proof).

Proposition 1. Let $M^{n+1}, n \geq 1$, be a Riemannian manifold endowed with a nontrivial field $X$ which is closed and conformal. Then we have that

a) The set $\mathcal{Z}(X)$ consisting of the points of $M^{n+1}$ where $X$ vanishes is a discrete set.

b) The unitary field $\mathcal{N}=X /|X|$ defined on the open dense set $M^{\prime}=M^{n+1}-$ $\mathcal{Z}(X)$ satisfies

$$
\nabla_{\mathcal{N}} \mathcal{N}=0 \quad \nabla_{u} \mathcal{N}=\frac{\phi}{|X|} \text { u if }\langle u, \mathcal{N}\rangle=0 .
$$

In particular, the flow of $\mathcal{N}$ is a unit speed geodesic flow.

c) The $n$-dimensional distribution $\mathcal{D}$ defined on $M^{\prime}$ by

$$
p \in M^{\prime} \longmapsto \mathcal{D}(p)=\left\{v \in T_{p} M^{n+1} \mid\langle X(p), v\rangle=0\right\}
$$

determines a codimension one umbilical Riemannian foliation $\mathcal{F}(X)$ which is oriented by $\mathcal{N}$. Moreover, the functions $|X|$ and $\phi$ are constant on connected leaves of $\mathcal{F}(X)$ and each leaf has constant mean curvature $\mathcal{H}=-\phi /|X|$. 
Understanding the interaction between the geometries of the manifold $M^{n+1}$ and that of leaves of the foliation $\mathcal{F}(X)$ will be important before studying hypersurfaces of $M$. For example, one can derive, directly from (2), that

$$
R(u, v) X=(u \phi) v-(v \phi) u \quad u, v \in T M,
$$

where $R$ is the curvature operator of $M$. From this, one has

$$
S(X, u)=-n u \phi \quad \text { that is } \operatorname{Ric}(X)=-n \nabla \phi,
$$

where $S$ and Ric are, respectively, the Ricci tensor and the Ricci operator of the metric on $M$. So, according to (5) and (7), the closed and conformal field $X$ is an eigenfield of the Ricci operator on the open set $M^{\prime}=M-\mathcal{Z}(X)$, corresponding to the eigenvalue $-n(X \phi) /|X|^{2}$.

Now, if we denote by $\mathcal{R}$ the curvature operator of the Riemannian induced structures on leaves of $\mathcal{F}(X)$, since these leaves are umbilical with constant mean curvature $\mathcal{H}=-\phi /|X|$, the Gauss equation gives us

$$
\mathcal{R}(u, v) w=R(u, v) w+\mathcal{H}^{2}(\langle v, w\rangle u-\langle u, w\rangle v),
$$

for all $u, v, w \in T \mathcal{F}(X)$. And so, if $\mathcal{S}$ represents the Ricci tensor of the metric induced on leaves,

$$
\mathcal{S}(u, u)=S(u, u)-R(u, \mathcal{N}, \mathcal{N}, u)+(n-1) \mathcal{H}^{2}|u|^{2} .
$$

Using (6), we may write

$$
|X|^{2} \mathcal{S}(u, u)=|X|^{2} S(u, u)+(n-1) \phi^{2}|u|^{2}+(X \phi)|u|^{2},
$$

for all $u \in T \mathcal{F}(X)$, that is, with $\langle u, X\rangle=0$.

We must point out that, because the flow of $\mathcal{N}$ transforms homothetically leaves of $\mathcal{F}(X)$, the curvature operator $\mathcal{R}$ is invariant through that flow and, as a consequence, the tensor $\mathcal{S}$ has this same property.

We have seen that the existence of a closed conformal field $X$ on our manifold $M^{n+1}$ gives a foliation $\mathcal{F}(X)$ by means of constant mean curvature (umbilical) hypersurfaces. The same pleasing condition on the geometry of $M$ that allowed us in $[\mathrm{M}]$ to prove some results of Liebmann and Alexandrov type implies here that compact leaves of the foliation $\mathcal{F}(X)$ are stable.

Proposition 2. Let $M^{n+1}, n \geq 1$, be a Riemannian manifold having a nontrivial closed conformal field $X$ and $\mathcal{F}(X)$ the corresponding umbilical foliation. Suppose that the direction determined by $X$ is the one of least Ricci curvature, that is,

$$
|X|^{2} S(u, u)-|u|^{2} S(X, X) \geq 0 \quad u \in T M^{n+1} .
$$


Then, each compact connected leaf of the foliation $\mathcal{F}(X)$ is a stable constant mean curvature hypersurface of $M^{n+1}$.

Proof. First of all, notice that the condition (10) on the Ricci tensor of $M$ can be rewritten, using (7) and (9), in this way

$$
|X|^{2} \mathcal{S}(u, u) \geq(n-1)\left(\phi^{2}-X \phi\right)|u|^{2} \quad \text { for all } u \in T \mathcal{F}(X) .
$$

Since, from Proposition $1,\left(\phi^{2}-X \phi\right) /|X|^{2}$ is a constant on each leaf of $\mathcal{F}(X)$, we can apply the Lichnerowicz theorem $([\mathrm{L}])$ on each compact leaf $\Sigma$ of the foliation and deduce

$$
\lambda_{1}(\Sigma) \geq n \frac{\phi^{2}-X \phi}{|X|^{2}}(\Sigma)
$$

where $\lambda_{1}$ stands for the first nonzero eigenvalue of the Laplacian of a compact Riemannian manifold. But, if $h \in C^{\infty}(\Sigma)$ is a smooth mean zero function, from definition (1) we have

$$
Q(h) \geq \int_{\Sigma}\left\{\lambda_{1}(\Sigma)-\left(S(N, N)+|\sigma|^{2}\right)\right\} h^{2} d A .
$$

Since $\Sigma$ is a leaf of $\mathcal{F}(X)$ we can take $N=\mathcal{N}$ and so, from (7),

$$
S(N, N)=-n \frac{X \phi}{|X|^{2}} \quad \text { is constant on } \Sigma .
$$

Also, as $\Sigma$ is umbilical, from Proposition 1 we obtain

$$
|\sigma|^{2}=n \mathcal{H}^{2}=n \frac{\phi^{2}}{|X|^{2}} \quad \text { also constant on } \Sigma \text {. }
$$

So, the above lower bound for $\lambda_{1}$ suffices to finish the proof.

\section{Riemannian warped products}

Suppose that $I$ denotes a 1-dimensional manifold (either circle or open interval of $\mathbb{R}$ ), that $P^{n}$ is an $n$-dimensional Riemannian manifold and that $f: I \rightarrow \mathbb{R}$ is a positive function. Then we can construct the so called warped product $I \times_{f} P$, which will be the $(n+1)$-dimensional differentiable manifold $I \times P$ endowed with the metric

$$
\langle,\rangle=\pi_{I}^{*}\left(d s^{2}\right)+\left(f \circ \pi_{I}\right)^{2} \pi_{P}^{*}\langle,\rangle_{P},
$$

where $\pi_{I}$ and $\pi_{P}$ are the projections from $I \times P$ on each factor and $\langle,\rangle_{P}$ is the metric on $P$. Also, we will write for simplicity

$$
\langle,\rangle=d s^{2}+f^{2}\langle,\rangle_{P} .
$$


One can easily check that the vector field

$$
X(s, q)=f(s)(\partial / \partial s)_{(s, q)} \quad s \in I \quad q \in P
$$

is a closed conformal nowhere vanishing field on this manifold. Moreover, the warped metric will be complete, for any $f$, if and only if $P$ is complete and $I$ is either a circle or the whole $\mathbb{R}$. It is noticeable that this is, basically, the only possible situation. In other words

If $M$ is a Riemannian manifold having a nontrivial closed and conformal field, then it is, near a point where the field does not vanish, locally isometric to a warped product with a 1-dimensional factor.

When our manifold $M^{n+1}$ having a non trivial closed conformal field is complete, we can globalize the assertion above. In fact, a more or less elaborated consequence of known facts (see $[\mathrm{M}]$ for a proof), is the following result.

Proposition 3. Let $M^{n+1}, n \geq 1$, be a complete Riemannian manifold having a non-trivial closed conformal field $X$. Then, $X$ has at most two zeroes and the following alternatives are the only possible ones, corresponding respectively to the cases where $X$ has one, two or no zeroes on $M^{n+1}$ :

a) $M^{n+1}$ is an Euclidean space with a rotationally invariant metric, that is, $M^{n+1}=\mathbb{R}^{n+1}$ and the metric, expressed in polar coordinates $(x=r p)$ on $\mathbb{R}^{n+1}$ $\{0\}=\mathbb{R}^{+} \times \mathbb{S}^{n}$, is, up to homotheties,

$$
d r^{2}+f(r)^{2} d \sigma_{n}^{2}
$$

where $d \sigma_{n}^{2}$ is the constant curvature one metric on the sphere $\mathbb{S}^{n}$ and $f$ is the positive restriction to $\mathbb{R}^{+}$of an odd differentiable function with $f^{\prime}(0)=1$. Moreover, the field $X$ is given by $X(r, p)=f(r) p$ for $r \in \mathbb{R}^{+}$and $p \in \mathbb{S}^{n} \subset \mathbb{R}^{n+1}$ and the leaves of the foliation $\mathcal{F}(X)$ are the spheres centered at the origin $r=r_{0}$.

b) $M^{n+1}$ is a sphere with a rotationally invariant metric, that is, $M^{n+1}=\mathbb{S}^{n+1}$ and the metric, expressed polar coordinates $(x=a \cos \theta+p \sin \theta)$ on $\mathbb{S}^{n+1}$ $\{a,-a\}=] 0, \pi\left[\times \mathbb{S}^{n}\right.$, where $a \in \mathbb{S}^{n+1}$ is arbitrary and $\mathbb{S}^{n}$ is the equator orthogonal to $a$, is given, up to homotheties, by

$$
d \theta^{2}+f(\theta)^{2} d \sigma_{n}^{2}
$$

where $f$ is the restriction to ]0, $\pi\left[\right.$ of a $2 \pi$-periodic odd function with $f^{\prime}(0)=1$ having no zeroes in $] 0, \pi[$. Moreover the field $X$ is $X(\theta, p)=f(\theta)(a \sin \theta-p \cos \theta)$, for each $\theta \in] 0, \pi\left[\right.$ and $p \in \mathbb{S}^{n} \subset \mathbb{S}^{n+1}$. In this case, the leaves of $\mathcal{F}(X)$ are the small spheres of $\mathbb{S}^{n+1}$ parallel to the equator $\mathbb{S}^{n}$.

c) The simply connected Riemannian covering of $M^{n+1}$ is a warped product $\mathbb{R} \times_{f} P^{n}$, where $P^{n}$ is a complete simply connected Riemannian $n$-dimensional 
manifold and $f$ is a positive function defined on $\mathbb{R}$. Moreover, the group $\Gamma$ of deck isometries is a subgroup of Iso $(\mathbb{R}) \times \operatorname{Iso}\left(P^{n}\right)$. In this case, the function $f$ is invariant by the translations in the projection of $\Gamma$ on $\operatorname{Iso}(\mathbb{R})$, the field $X$ is determined by the projection of $f(s)(\partial / \partial s)_{(s, p)}$, for any $s \in \mathbb{R}$ and $p \in P^{n}$, and the foliation $\mathcal{F}(X)$ consists of the projections of the slices $\{s\} \times P^{n}, s \in \mathbb{R}$.

Remark 1. Proposition 3 describes all complete manifolds $M^{n+1}$ carrying a nontrivial closed conformal field as either a quotient of a warped product (in the case $c)$ ) or as a warped product completed with one or two points (in the cases $a$ ) and $b$ ) of rotationally invariant metrics on Euclidean or spherical spaces). Let $I \times_{f} P^{n}$ be the warped product appearing in each case. Then, Proposition 3 asserts that the geometry of $M$ and that of the foliation $\mathcal{F}(X)$ is completely described by means of the geometry of a standard leaf of the foliation $\mathcal{F}(X)$, that is, the geometry of $P$, and by the warping function $f$. The corresponding translation between these two languages can be easily done. For example, the function $|X|$ is now $f \circ \pi_{I}$ (for the sake of simplicity, we will omit the projections and write $f$ ); the function $\phi$ appearing in (2) is the derivative $f^{\prime}$; the mean curvature function $\mathcal{H}$ of leaves becomes - $(\log f)^{\prime}$; vectors tangent to each leaf of the foliation, that is, vectors in $T \mathcal{F}(X)$ are canonically identified with vectors tangent to $P$; and, finally, one can also see that, with this identification, equation (8) is now

$$
S(u, v)=S_{P}(u, v)-\left(f f^{\prime \prime}+(n-1) f^{\prime 2}\right)\langle u, v\rangle_{P} \quad u, v \in T P .
$$

Thus, for instance, $M$ has constant sectional curvature $\bar{c}$ if and only if (see [On], p. 345) $P$ has constant sectional curvature $c$ and $f$ is a positive solution of the equation $f^{\prime 2}+\bar{c} f^{2}=c$.

We said in Section 2 that a non-trivial closed conformal field $X$ on a Riemannian manifold $M^{n+1}$ is always an eigenfield of the Ricci operator corresponding to the eigenvalue $-n(X \phi) /|X|^{2}$, away from its zeroes. Ambient spaces where we will be able to classify all stable constant mean curvature hypersurfaces are those Riemannian manifolds where this eigenvalue is constant. That is, when

$$
S(X, X)=n \kappa|X|^{2} \quad \text { or } X \phi=-\kappa|X|^{2} \quad \text { for some } \kappa \in \mathbb{R} .
$$

If this occurs, then, from (5), we obtain

$$
\nabla \phi=-\kappa X
$$

And, so, from (2), the function $\phi$ satisfies an Obata type equation

$$
\nabla^{2} \phi+\kappa \phi\langle,\rangle=0 .
$$

From Proposition 3, such $M$ is either a warped product completed with one or two points (when it is a rotationally invariant Euclidean or spherical space) or it 
is a quotient of a warped product. In any of these two cases the warping function $f$ which appears in Proposition 3 is, according to Remark 1 and taking (12) into account, a solution of the Jacobi equation

$$
f^{\prime \prime}+\kappa f=0
$$

If our manifold is in the case a) of Proposition 3 , that is, if it is $\mathbb{R}^{n+1}$ with a rotationally invariant metric, we have that $f$ only vanishes at 0 , so $\kappa \leq 0$, and either $f(r)=\lambda r$ for some $\lambda \in \mathbb{R}-\{0\}$ or $f(r)=\sinh \sqrt{-\kappa} r$ for each $r \in \mathbb{R}^{+}$, according as $\kappa=0$ or $\kappa<0$. Then $M$ is either an Euclidean or a hyperbolic space. Analogously, in the case b) of Proposition 3, we have $\kappa>0$ and $f(\theta)=\sin \sqrt{\kappa} \theta$ for $\theta \in \mathbb{R}$. Hence, in this case, $M$ is a spherical space. Finally, if $M$ is in the case c) of this same Proposition 3, then the warping function $f$ of its simply connected Riemannian covering must be constant if $\kappa=0$ and, if $\kappa<0$, it must be, up to changes of origin in $\mathbb{R}$, one of these two functions

$$
f(s)=\cosh \sqrt{-\kappa} s \quad f(s)=e^{\sqrt{-\kappa} s} \quad s \in \mathbb{R} .
$$

Since these two functions are not invariant through any isometry of $\mathbb{R}$, our manifold must be, up to homotheties, a quotient of a Riemannian product $\mathbb{R} \times P^{n}$ (and, in this case, the field $X$ must be parallel), or a warped product $\mathbb{R} \times_{e^{s}} P^{n}$, or finally, a warped product $\mathbb{R} \times_{\cosh s} P^{n}$. These last two types of Riemannian manifolds have been sometimes refered to as pseudo-hyperbolic spaces (see $[\mathrm{Ta}]$ ). Then we may state the following

Proposition 4. Let $M^{n+1}, n \geq 1$, a complete Riemannian manifold with a nonparallel closed conformal field $X$ such that the direction determined by $X$ has constant Ricci curvature. Then $M^{n+1}$ is either a Euclidean space or a sphere or, up to homotheties, a warped product $\mathbb{R} \times e^{s} P^{n}$ or a warped product $\mathbb{R} \times{ }_{\cosh s} P^{n}$, being $P^{n}$ any complete Riemannian manifold.

Remark 2. It would be interesting to remark that, among all the possible pseudo-hyperbolic spaces that we can find in Proposition 4 above, there are some of them specially important: those where $P^{n}$ is chosen to be a flat or hyperbolic space form. That is

$$
\mathbb{R} \times e^{s}\left(\mathbb{R}^{n} / \Gamma\right) \quad \mathbb{R} \times \cosh s\left(\mathbb{H}^{n} / \Psi\right),
$$

where $\Gamma$ is a torsion-free crystallographic group and $\Psi$ is a group of hyperbolic transformations acting properly and discontinuously, giving an orientable quotient. These quotients can be also viewed as

$$
\left(\mathbb{R} \times e^{s} \mathbb{R}^{n}\right) /\left(I_{\mathbb{R}} \times \Gamma\right) \quad\left(\mathbb{R} \times \cosh s \mathbb{H}^{n}\right) /\left(I_{\mathbb{R}} \times \Psi\right) .
$$

But it is immediate to check that $\mathbb{R} \times e^{s} \mathbb{R}^{n}$ and $\mathbb{R} \times \cosh s \mathbb{H}^{n}$ are respectively isometric to the hyperbolic space $\mathbb{H}^{n+1}$. Hence, these two types of warped products appearing in Proposition 4 are hyperbolic space forms. Recall also that the ends of complete three dimensional hyperbolic space forms are all of the form $]-\infty, a] \times{ }_{e^{s}}$ $\mathbb{T}^{2}$, where $a \in \mathbb{R}$ and $\mathbb{T}^{2}$ is a flat two torus. 


\section{Minkowski formulae}

Consider now an orientable compact hypersurface $\Sigma^{n}$ immersed into our Riemannian manifold $M^{n+1}$ endowed with a non-trivial closed conformal field $X$ and, so, with the corresponding umbilical foliation $\mathcal{F}(X)$. Denote by $\operatorname{div}_{\Sigma}$ the divergence of vector fields tangent to $\Sigma$ and by $X^{T}$ the component of $X$ tangent to $\Sigma$, that is $X^{T}=X-\langle X, N\rangle N$, where $N$ is a unit normal field to the immersion. From (2), it is clear that $\operatorname{div}_{\Sigma} X^{T}=n \phi+n H\langle X, N\rangle$. Hence

$$
\int_{\Sigma}\{\phi+H\langle X, N\rangle\} d A=0
$$

where $H$ is the mean curvature function corresponding to that choice of orientation and $d A$ is the Riemannian measure induced on $\Sigma$. This integral formula should be called first Minkowski formula for $\Sigma$ because, when the manifold is the Euclidean space $\mathbb{R}^{n+1}$ and the field $X$ is the position vector field, it yields the so-called classical formula, which has been traditionally used to obtain rigidity theorems for hypersurfaces. Analogous formulae have been derived when the manifold $M$ is another simply connected space form, that is, $M=\mathbb{S}^{n+1}$ or $M=\mathbb{H}^{n+1}$ (see $[\mathrm{Biv}, \mathrm{MR}])$.

In $[\mathrm{M}]$, we used a second Minkowski formula obtained from the first one, by following the same method as we had already made in $[\mathrm{MR}]$ where we dealt with the case of simply connected space forms. We include here a sketch in order to make the paper selfcontained. In fact, if $\psi: \Sigma \rightarrow M$ is our immersion, we know that, for $s$ close enough to zero, the parallel map $\psi_{s}: \Sigma \rightarrow M$ given by $\psi_{s}(p)=\exp _{\psi(p)} s N(p), p \in \Sigma$ is another immersion and $\Sigma_{s}=\psi_{s}(\Sigma)$ is called the parallel hypersurface at distance $s$. For each small $s$, we have a corresponding first Minkowski formula (13) for the hypersurface $\Sigma_{s}$. In fact, we have

$$
\int_{\Sigma}\left\{\phi \circ \psi_{s}+H_{s}\left\langle X \circ \psi_{s}, N_{s}\right\rangle\right\} d A_{s}=0
$$

where

$$
N_{s}(p)=\frac{d}{d s} \exp _{\psi(p)} s N(p) \quad p \in \Sigma,
$$

is a unit field normal to the immersion $\psi_{s}, H_{s}$ is the mean curvature of $\psi_{s}$ with respect to $N_{s}$ and $d A_{s}$ the Riemannian measure induced on $\Sigma_{s}$. Now, we derive with respect to the variable $s$ at $s=0$ in this equality. Recalling that the mean curvature of hypersurfaces is the gradient of the induced Riemannian measure, that is,

$$
\left.\frac{d}{d s}\right|_{s=0} d A_{s}=-n H d A
$$

we have

$$
\int_{\Sigma}\left\{\left.\frac{d}{d s}\right|_{s=0}\left[\phi \circ \psi_{s}+H_{s}\left\langle X \circ \psi_{s}, N_{s}\right\rangle\right]-n H[\phi+H\langle X, N\rangle]\right\} d A=0 .
$$


On the other hand, using (7), we obtain

$$
\left.\frac{d}{d s}\right|_{s=0} \phi \circ \psi_{s}=\langle\nabla \phi, N\rangle=-\frac{1}{n} S(X, N) .
$$

In the same way, using (2) and recalling that $N_{s}(p)$ is the tangent field along the geodesic $\psi_{s}(p)$, for each $p \in \Sigma$, we get

$$
\left.\frac{d}{d s}\right|_{s=0}\left\langle X \circ \psi_{s}, N_{s}\right\rangle=\left\langle\nabla_{N} X, N\right\rangle=\phi
$$

Moreover the Weingarten map $A_{s}$ of the immersion $\psi_{s}$, defined by $A_{s} u=-\nabla_{u} N_{s}$, for each $u$ tangent to $\Sigma_{s}$, satisfies a Ricatti evolution equation

$$
\frac{d}{d s} A_{s}-A_{s}^{2}-R_{N_{s}}=0
$$

where $R_{N_{s}}$ is the curvature operator of $M$ in the direction of $N_{s}$. This is a consequence from (2) and the Ricci identities on $M$. This, or a direct computation, gives

$$
\left.\frac{d}{d s}\right|_{s=0} H_{s}=\frac{1}{n}\left\{|\sigma|^{2}+S(N, N)\right\},
$$

where $\sigma$ denotes the second fundamental form of $\Sigma$. Substituting the three equalities above in the last integral formula, one has

$$
\int_{\Sigma}\left\{n(n-1)\left[H \phi+H_{2}\langle X, N\rangle\right]+S(X, N)-S(N, N)\langle X, N\rangle\right\} d A=0
$$

where $H_{2}$ is defined by the equality $n(n-1) H_{2}=n^{2} H^{2}-|\sigma|^{2}$, that is, $H_{2}$ is the normalized second elementary symmetric polynomial in the principal curvatures of $\Sigma$ (see, for example, $[\mathrm{MR}]$ ). We are going to modify slightly this integral formula. Since the zero set $\mathcal{Z}(X)$ is discrete, there is only a finite number of zeroes of $X$ on the compact hypersurface $\Sigma$. Then, the unit field $\mathcal{N}$ is defined on $\Sigma$ except a finite number of points and we have that $X$ is an eigenvector of the Ricci operator on $\Sigma-\mathcal{Z}(X)$. So

$$
S(X, N)=S(\mathcal{N}, \mathcal{N})\langle X, N\rangle
$$

is true unless for a finite number of points. Using this fact, we finally may write the second Minkowski formula for the compact hypersurface $\Sigma$.

$$
\int_{\Sigma}\left\{n(n-1)\left[H \phi+H_{2}\langle X, N\rangle\right]+[S(\mathcal{N}, \mathcal{N})-S(N, N)]\langle X, N\rangle\right\} d A=0
$$

which is significative only when $n>1$. 


\section{Stability results}

Since we have all the necessary preliminaries, let us consider the geometric situation being the main purpose of this paper. Let $\Sigma^{n}$ be a stable constant mean curvature compact orientable hypersurface immersed in a Riemannian manifold $M^{n+1}, n \geq 1$, endowed with a nontrivial closed conformal field $X$. Using the first Minkowski formula (13), we can see that $h \in C^{\infty}(\Sigma)$ defined by

$$
h=\phi_{\mid \Sigma}+H\langle X, N\rangle,
$$

is a mean zero function, where $N$ is a unit normal field on $\Sigma$ and $H$ the corresponding (constant) mean curvature. Then, because the stability of $\Sigma$, we can use it as a test function in (2).

Take a vector $u \in T \Sigma$ tangent to the hypersurface. Then, according to Proposition 1 , as $\phi$ is locally constant with respect to the directions orthogonal to the field $X$, we have

$$
u \phi_{\mid \Sigma}=\frac{X \phi}{|X|^{2}}\langle u, X\rangle \text {. }
$$

Suppose now that the field $X$ is in the conditions of Proposition 4, that is, that $\operatorname{Ric}(X)=n \kappa X$ or $X \phi=-\kappa|X|^{2}$ for some $\kappa \in \mathbb{R}$. Then, taking derivatives again with respect to another tangent vector $v \in T \Sigma$, we obtain

$$
\left(\nabla^{2} \phi_{\mid \Sigma}\right)(u, v)=-\kappa(\langle A u, v\rangle\langle X, N\rangle+\phi\langle u, v\rangle),
$$

where $A$ is the Weingarten map of the hypersurface associated to the unit field $N$. In a similar way, we can compute the Hessian of the function $\langle X, N\rangle$ getting

$$
\left(\nabla^{2}\langle X, N\rangle\right)(u, v)=-\phi\langle A u, v\rangle-\left\langle X,\left(\nabla_{v} A\right) u\right\rangle-\langle A u, A v\rangle\langle X, N\rangle,
$$

for all $u, v \in T \Sigma$. Taking traces in both (15) and (16) and using the Codazzi equation and (7), we have

$$
\Delta h=-n \kappa \phi-n H^{2} \phi-H\langle X, N\rangle S(N, N)-H|\sigma|^{2}\langle X, N\rangle .
$$

Now, we can evaluate the selfadjoint operator associated to the index form $Q$ over the function $f$ and obtain

$$
\left(\Delta+S(N, N)+|\sigma|^{2}\right) h=\left(|\sigma|^{2}-n H^{2}+S(N, N)-n \kappa\right) \phi .
$$

Hence, we conclude finally that

$$
\begin{aligned}
Q(h) & =-\int_{\Sigma} h\left(\Delta+S(N, N)+|\sigma|^{2}\right) h d A \\
& =-\int_{\Sigma}\left(|\sigma|^{2}-n H^{2}+S(N, N)-n \kappa\right) \phi(\phi+H\langle X, N\rangle) d A .
\end{aligned}
$$


On the other hand, since the mean curvature $H$ of $\Sigma$ is constant, we can combine the two Minkowski formulae (13) and (14) and take into account that $S(\mathcal{N}, \mathcal{N})=$ $n \kappa$ to deduce that

$$
\int_{\Sigma}\left\{|\sigma|^{2}-n H^{2}+S(N, N)-n \kappa\right\}\langle X, N\rangle d A=0
$$

Substituting this equation in the right side of (17) and recalling that our hypersurface $\Sigma$ is stable (1), we finally have

$$
0 \leq Q(h)=-\int_{\Sigma}\left\{|\sigma|^{2}-n H^{2}+S(N, N)-n \kappa\right\} \phi^{2} d A
$$

From this inequality we will argue to classify the stable constant mean curvature compact orientable hypersurfaces of a wide set of Riemannian manifolds including simply connected space forms and, also, many nonsimply connected space forms and manifolds having nonconstant sectional curvature.

Theorem 5. Let $M^{n+1}, n \geq 1$, be a Riemannian manifold with a non parallel closed conformal field $X$ whose direction is the one of the least Ricci curvature $n \kappa$, $\kappa \in \mathbb{R}$. Moreover, suppose that the Ricci curvature in that direction is constant. Then, each connected component of a stable constant mean curvature compact orientable hypersurface of $M^{n+1}$ is either an umbilical round sphere or a leaf of the corresponding foliation $\mathcal{F}(X)$. When the first case holds $M^{n+1}$ must have constant sectional curvature $\kappa$ near to that connected component.

Proof. Firstly notice that, from our hypothesis, we have the inequality (18) on our stable hypersurface. Now, let us see that those same hypothesis imply that the integrand in (18) is nonnegative. In fact, we have that $|\sigma|^{2}-n H^{2} \geq 0$ from the Schwarz inequality. On the other hand, since the inequality (10) holds and $S(X, X)=n \kappa|X|^{2}$ with $\kappa \in \mathbb{R}$, we have $S(N, N)-n \kappa \geq 0$. Thus, the stability assumption implies that

$$
|\sigma|^{2}-n H^{2}=0 \quad \text { and } \quad S(N, N)=n \kappa,
$$

because $\phi=f^{\prime} \circ \pi_{\mathbb{R}}$ only vanishes on isolated leaves of the corresponding foliation, being $X$ nonparallel. That is, our hypersurface is umbilical and its normal direction attains the least value of the Ricci curvature of the ambient space $M$. From the first of these two assertions we have that, if $u \in T \Sigma$ is a vector tangent to the hypersurface,

$$
u\langle X, N\rangle=-H\langle X, u\rangle .
$$

Then, we see that the function $H \phi-\kappa\langle X, N\rangle$ is constant on each connected component of $\Sigma$. Let us work on such a connected component $\Sigma_{0}$. So, we suppose 
$H \phi-\kappa\langle X, N\rangle=d$ with $d \in \mathbb{R}$ on $\Sigma_{0}$. From this, (15), (16) and recalling that $\Sigma_{0}$ is umbilical, we have

$$
\nabla^{2} \phi=-\left\{\left(\kappa+H^{2}\right) \phi-H d\right\}\langle,\rangle \quad \nabla^{2}\langle X, N\rangle=-\left\{\left(\kappa+H^{2}\right)\langle X, N\rangle+d\right\}\langle,\rangle .
$$

From these two equations, using Theorem 2 of [Ta], and recalling that $\Sigma_{0}$ is compact, we can conclude that either $\Sigma_{0}$ is a sphere with constant curvature $\kappa+H^{2}>0$ or these two functions are constant and, so, either $X^{T}=0$, that is, the field $X$ is normal to our hypersurface and it must be a leaf of the foliation $\mathcal{F}(X)$, or $\kappa=H=0$. So, it only remains to analyze the two cases when $\Sigma_{0}$ is a round sphere with curvature $\kappa+H^{2}$ and the case $\kappa=H=0$.

In the first one, we have that, if $R_{\Sigma_{0}}$ is the curvature operator of the induced metric on the hypersurface $\Sigma_{0}$, then $R_{\Sigma_{0}}=\left(\kappa+H^{2}\right) R_{0}$, where $R_{0}$ represents the curvature operator of a constant one curvature space. Hence, taking into account the Gauss equation for the immersion of $\Sigma$ in $M$, and the fact that this immersion is totally umbilical,

$$
R(u, v) w=R_{\Sigma}(u, v) w-H^{2} R_{0}(u, v) w=\kappa R_{0}(u, v) w \quad u, v, w \in T \Sigma_{0} .
$$

This last equation and the fact that

$$
R(u, v) X=\kappa\{\langle v, X\rangle u-\langle u, X\rangle v\}=\kappa R_{0}(u, v) X \quad u, v \in T \Sigma_{0},
$$

which is a consequence from (5) and (6), give us (notice that $X$ is transverse to $\Sigma_{0}$ unless perhaps on a zero measure set)

$$
R=\kappa R_{0} \quad \text { at the points of } \Sigma_{0} .
$$

Now, using the Gauss equation (8) for the foliation $\mathcal{F}(X)$, one has

$$
\mathcal{R}(u, v) w=\left(\kappa+\mathcal{H}^{2}\right) R_{0}(u, v) w \quad u, v, w \in T_{p} \mathcal{F}(X) \quad p \in \Sigma_{0} .
$$

But, since the operator $\mathcal{R}$ is invariant through the flow of $\mathcal{N}$, as we had pointed out at the end of Section 2, we obtain that

$$
\mathcal{R}=\left(\kappa+\mathcal{H}^{2}\right) R_{0} \quad \text { on } \Omega,
$$

being $\Omega$ a certain neighborhood of $\Sigma_{0}$. We use again the Gauss equation (8) and see that

$$
R(u, v) w=\kappa R_{0}(u, v) w \quad u, v, w \in T_{x} \mathcal{F}(X) \quad x \in \Omega .
$$

Hence, as $X$ is orthogonal to $\mathcal{F}(X)$ and we have (19), we deduce that $R=\kappa R_{0}$ on $\Omega$, that is, $\Omega$ has near to $\Sigma_{0}$ constant curvature equal to $\kappa$.

In the second case we suppose $\kappa=H=0$. Then, from the discussion before Proposition 4, we have that $\phi$ is constant on $M$. But, using the first Minkowski 
formula (13), we conclude that $\phi$ is identically zero and, so, $X$ would be parallel. This is not possible according to our hypothesis.

We know from Section 3 that, if $I$ is an open interval of $\mathbb{R}$ and $f \in C^{\infty}(I)$ is a positive function, the warped product $I \times_{f} P^{n}$, where $P^{n}$ is any Riemannian manifold, is a $(n+1)$-manifold such that $X=\left(f \circ \pi_{I}\right)(\partial / \partial s)$ is a non trivial closed conformal field, which is nonparallel provided that $f$ is nonconstant. From Remark 1, one can see that the Ricci curvature of $I \times_{f} P^{n}$ attains its minimum in the direction of $X$ if and only if

$$
S_{P} \geq(n-1)\left(f^{\prime 2}-f f^{\prime \prime}\right)\langle,\rangle_{P},
$$

where $S_{P}$ and $\langle,\rangle_{P}$ are respectively the Ricci and the metric tensors of $P^{n}$. From that same Remark 1, one deduces that the Ricci curvature in the direction of $X$ is constant if and only if the function $f$ is a solution to the Jacobi equation

$$
f^{\prime \prime}+\kappa f=0 \quad \text { for some } \kappa \in \mathbb{R} .
$$

In this case, notice that

$$
f^{\prime 2}-f f^{\prime \prime}=f^{\prime 2}+\kappa f^{2} \quad \text { is a constant. }
$$

Finally, if one recalls that the leaves of the foliation $\mathcal{F}(X)$ are, in this situation, the slices $\{s\} \times P^{n}$, one obtains the following consequence of Theorem 5 .

Corollary 6. Let $f$ be a positive nonconstant solution of $f^{\prime \prime}+\kappa f=0, \kappa \in \mathbb{R}$ defined on an open interval $I$ and let $P^{n}$ be a Riemannian manifold whose Ricci curvature satisfies $S_{P} \geq(n-1)\left\{{f^{\prime}}^{2}+\kappa f^{2}\right\}$. Suppose that $\Sigma^{n}$ is a stable constant mean curvature compact orientable hypersurface in the warped product $I \times_{f} P^{n}$. Then each connected component of $\Sigma^{n}$ is either an umbilical round sphere (and, in this case, $\pi_{P}\left(\Sigma^{n}\right) \subset P^{n}$ would have constant curvature $\kappa$ ) or a slice $\{s\} \times P^{n}$, for some $s \in I$. Moreover, there is at most one component of the first type and, when $\kappa \geq 0$ the hypersurface $\Sigma^{n}$ is necessarily connected.

Proof. It only remains to prove the two last assertions concerning the connectedness of $\Sigma$. Firstly, notice that, if $\Sigma$ has one connected component $\Sigma_{1}$ of the first type, since $S(N, N)=n \kappa$ because $M$ has constant curvature $\kappa$ near to $\Sigma$ and $|\sigma|^{2}=n H^{2}$ because $\Sigma_{1}$ is umbilical, the index form $Q_{\mid \Sigma_{1}}$ becomes

$$
Q_{\mid \Sigma_{1}}(u)=\int_{\Sigma_{1}}\left\{|\nabla u|^{2}-n\left(\kappa+H^{2}\right) u^{2}\right\} d A .
$$

So, as $\kappa+H^{2}>0$, this quadratic form $Q_{\mid \Sigma_{1}}$ has index one on the space $C^{\infty}\left(\Sigma_{1}\right)$. Then, if $\Sigma$ is stable, all the remaining connected components of $\Sigma$ must be of 
the second type. Let $\Sigma_{2}$ be such a component. As the least value of the Ricci curvature is $n \kappa=-n f^{\prime \prime} / f$, we have

$$
Q_{\mid \Sigma_{2}}(u)=\int_{\Sigma}\left\{|\nabla u|^{2}+n\left(\frac{f^{\prime \prime}}{f}-\mathcal{H}^{2}\right) u^{2}\right\} d A
$$

for all $u \in C^{\infty}\left(\Sigma_{2}\right)$. But

$$
\mathcal{H}=-\frac{\phi}{|X|}=-\frac{f^{\prime}}{f} \circ \pi_{I}
$$

If we have also that $\kappa \geq 0$, then ${f^{\prime}}^{2}+\kappa f^{2}>0$ and $Q_{\mid \Sigma_{2}}$ has also index one, as desired.

When one requires the ambient space $M^{n+1}$ in Theorem 5 to be complete, Proposition 4 lets us reduce to consider Euclidean spaces, spheres or the so called pseudo-hyperbolic spaces. Then, one has the following new classification results.

Corollary 7. Let $\Sigma^{n}$ be a stable orientable compact hypersurface with constant mean curvature immersed in the warped product $\mathbb{R} \times \cosh s P^{n}$, where $P^{n}$ is any complete Riemannian manifold whose Ricci curvature satisfies $S_{P} \geq-(n-1)$. Then, each connected component of $\Sigma^{n}$ must be either an umbilical round sphere (and, in this case, $P^{n}$ is a hyperbolic space form near $\pi_{P}\left(\Sigma^{n}\right)$ ) or a slice $\{s\} \times P^{n}$, $s \in \mathbb{R}$, (and, in this case, $P^{n}$ is compact). Moreover, there exists at most one component of the first type.

Remark 3. If one chooses $P^{n}$ in Corollary 7 above to be an orientable hyperbolic space form, that is, $P^{n}=\mathbb{H}^{n} / \Gamma$ where $\Gamma$ is a subgroup of Iso ${ }^{+}\left(\mathbb{H}^{n}\right)$ acting properly and discontinuously, then the corresponding ambient space $\mathbb{R} \times \cosh s P^{n}$ is, according to Remark 2 , an orientable hyperbolic space form, which can be easily identified with a quotient $\mathbb{H}^{n+1} / \Psi$, where $\Psi$ is a subgroup of Iso ${ }^{+}\left(\mathbb{H}^{n+1}\right)$ fixing a given hyperbolic hyperplane.

Corollary 8. Let $\Sigma^{n}$ be an orientable compact hypersurface with constant mean curvature immersed in the warped product $\mathbb{R} \times_{e^{s}} P^{n}$, where $P^{n}$ is any complete Riemannian manifold with nonnegative Ricci curvature. Then, $\Sigma^{n}$ is stable if and only if it is either an umbilical round sphere (and, in this case, $P^{n}$ is a flat space form near $\pi_{P}\left(\Sigma^{n}\right)$ ) or a finite number of slices $\{s\} \times P^{n}, s \in \mathbb{R}$ (and, in this case, $P^{n}$ is compact).

Proof. It only remains to see that it is not possible, with that ambient space, to have one connected component $\Sigma_{1}$ of the first class, that is, an umbilical round sphere, and another one $\Sigma_{2}$ of the second type, that is, a slice $\{s\} \times P^{n}$. Reasoning by contradiction, suppose that this is the case. Then, we already know that the index form $Q_{\mid \Sigma_{1}}$ has index one on the space $C^{\infty}\left(\Sigma_{1}\right)$. On the other hand, the index form $Q_{\mid \Sigma_{2}}$ is nonnegative on the whole $C^{\infty}\left(\Sigma_{2}\right)$ because $S(N, N)=S(\mathcal{N}, \mathcal{N})=$ 


$$
\begin{array}{r}
-n \kappa=-n \text { and }|\sigma|^{2}=n \mathcal{H}^{2}=n\left(f^{\prime} / f\right)^{2}=n \text { and, so, } \\
Q_{\mid \Sigma_{2}}(h)=\int_{\Sigma_{2}}|\nabla h|^{2} d A,
\end{array}
$$

for all $h \in C^{\infty}\left(\Sigma_{2}\right)$. This suffices to find a contradiction with the stability of $\Sigma$. Moreover, this same equality proves that any finite number of slices is a stable constant mean curvature hypersurface.

Remark 4. If we put in Corollary 8 above $P^{n}=\mathbb{R}^{n} / \Gamma$ where $\Gamma$ is a subgroup of Iso ${ }^{+}\left(\mathbb{R}^{n}\right)$ acting properly and discontinuously, then the corresponding ambient space $\mathbb{R} \times e_{e^{s}} P^{n}$ is, according to Remark 2, an orientable hyperbolic space form, which can be easily identified with a quotient $\mathbb{H}^{n+1} / \Psi$, where now $\Psi$ is a subgroup of Iso ${ }^{+}\left(\mathbb{H}^{n+1}\right)$ fixing a given horosphere.

One of the possible strategies to solve the isoperimetric problem in some Riemannian manifolds is to find all stable compact constant mean curvature hypersurfaces. The reason is that, at least in the low dimensional cases, domains which are solutions to that problem have as boundary smooth constant mean curvature hypersurfaces which are stable (see, for example, [RR1]). In our situation, Corollary 8 could be useful to solve the isoperimetric problem in $\mathbb{R} \times_{e^{s}} P^{n}$, where $P^{n}$ is a compact Riemannian manifold (this would include, for example, the ends of complete three dimensional hyperbolic manifolds). But there is a shorter way in order to get it, which also works without restrictions on the Ricci curvature of $P^{n}$. In fact, let $\Omega$ be any finite volume domain in that manifold $\mathbb{R} \times{ }_{e^{s}} P^{n}$, whose boundary is a compact hypersurface $\Sigma$. We represent by $F_{t}, t \in \mathbb{R}$, the flow of the corresponding closed conformal field $X$ and by $V(t)$ and $A(t)$ the $(n+1)$ dimensional volume of the domain $\Omega_{t}=F_{t}(\Omega)$ and the $n$-dimensional volume of the hypersurface $\Sigma_{t}=F_{t}(\Sigma)$. Then

$$
V^{\prime}(t)=\int_{\Omega_{t}} \operatorname{div} X=-\int_{\Sigma_{t}}\langle X, N\rangle d A \geq \int_{\Sigma_{t}}|X| d A
$$

from the Schwarz inequality. But, in our case, $|X|(s, q)=\phi(s, q)=e^{s}$ for any $(s, q) \in \mathbb{R} \times P$. So, using also the first Minkowski formula (13), we have

$$
V^{\prime}(t) \geq \int_{\Sigma_{t}} \phi d A=-\int_{\Sigma_{t}} H\langle X, N\rangle d A
$$

Now, if one recalls that the first variation of the area formula says that

$$
A^{\prime}(t)=-n \int_{\Sigma_{t}} H\langle X, N\rangle d A
$$

one can finally deduce that

$$
n V^{\prime}(t) \geq A^{\prime}(t) \quad \text { for each } t \in \mathbb{R},
$$


and the equality holds if and only if all the connected components of $\Sigma$ are slices $\{s\} \times P$. Integrating in this inequality and taking into account that, in this case,

$$
\lim _{t \rightarrow-\infty} A(t)=\lim _{t \rightarrow-\infty} V(t)=0
$$

we obtain that,

Proposition 9. If $\Sigma^{n}$ is a compact hypersurface of $\mathbb{R} \times_{e^{s}} P^{n}$ bounding a finite volume domain $\Omega^{n+1}$, we have that

$$
n \text { Volume } \Omega \geq \text { Area } \Sigma,
$$

and the equality occurs if and only if $\Sigma^{n}$ consists of an even number of slices $\{s\} \times P^{n}$.

Notice that this isoperimetric inequality was obtained by Yau in [Y] for domains in hyperbolic spaces, that is, when $P^{n}=\mathbb{R}^{n}$ is the Euclidean space (see Remark 2 ), but that, in his case, the equality is never attained. An analogue also exists for domains of minimal submanifolds in hyperbolic space (see [CG]). From this Proposition 9 and after an easy computation about the most favourable number of connected components of the hypersurface $\Sigma=\partial \Omega$, we conclude that

The solutions to the isoperimetric problem in a warped product $\mathbb{R} \times_{e^{s}} P^{n}$, where $P^{n}$ is any compact Riemannian manifold are of the form $\left.]-\infty, a\right] \times P^{n}$, for each $a \in \mathbb{R}$.

\section{References}

[A] A.D. Alexandrov, Uniqueness theorems for surfaces in the large I, Vestnik Leningrad Univ. 11 (1956), 5-17.

[BdC] J.L. Barbosa and M.P. do Carmo, Stability of hypersurfaces with constant mean cuvature, Math. Z. 185 (1984), 339-353.

[BdCE] J.L. Barbosa, M.P. do Carmo and J. Eschenburg, Stability of hypersurfaces with constant mean curvature in Riemannian manifolds, Math. Z. 197 (1988), 123-138.

[BiOn] R.L. Bishop and B. O'Neill, Manifolds of negative curvature, Trans. Amer. Math. Soc. 145 (1969), 1-49.

[Biv] I. Bivens, Integral formulas and hyperspheres in a simply connected space form, Trans. Amer. Math. Soc. 88 (1983), 113-118.

[CG] J. Choe and R. Gulliver, Isoperimetric inequalities on minimal submanifolds of space forms, Manuscripta Math. $\mathbf{7 7}$ (1992), 169-189.

[EI] A. El Soufi and S. Ilias, Majoration de la seconde valeur propre d'un operateur de Schrödinger sur une variété compacte et applications, J. Funct. Anal. 103 (1992), 294316.

[H] E. Heintze, Extrinsic upper bounds for $\lambda_{1}$, Math. Ann. 280 (1988), 389-402. 
[J] J.J. Jellett, Sur la surface dont la courbure moyenne est constante, J. Math. Pures Appl. 18 (1853), 163-167.

[K] M. Kanai, On a differential equation characterizing a Riemannian structure on a manifold, Tokyo J. Math. 6 (1983), 143-151.

[L] A. Lichnerowicz, Géométrie des Groupes des Transformations, Dunod, Paris, 1958.

[Li] H. Liebmann, Eine neue Eigenschaft der Kugel, Nachr. Kg. Ges. Wiss. Götingen, Math. Phys. Kl. (1899), 44-55.

[M] S. Montiel, Unicity of constant mean curvature hypersurfaces in foliated Riemannian manifolds, preprint.

[MR] S. Montiel and A. Ros, Compact hypersurfaces: the Alexandrov theorem for higher order mean curvatures, pp. 279-296. In: B. Lawson and K. Tenenblat (Eds.): Pitman Monographs and Surveys, in honor to M. P. Carmo, Pure and Applied Mathematics 52 (1991).

[O] M. Obata, Conformal transformations of Riemannian manifolds, J. Diff. Geom. 4 (1970), 311-333.

[On] B. O'Neill, Semi-Riemannian Geometry, Academic Press, New York 1983.

[RR1] M. Ritoré and A. Ros, Stable constant mean curvature tori and the isoperimetric problem in three space forms, Comment. Math. Helv. 67 (1992), 293-305.

[RR2] M. Ritoré and A. Ros, The spaces of index one minimal surfaces and stable constant mean curvature surfaces embedded in flat three manifolds, Trans. Amer. Math. Soc. 348 (1996), 391-410.

[RU] A. Ros and F. Urbano, Lagrangian submanifolds of $\mathbb{C}^{n}$ with conformal Maslov form and the Whitney sphere, J. Math. Soc. Japan. 50 (1) (1998), 203-226.

[TW] S. Tanno and W. Weber, Closed conformal vector fields, J. Diff. Geom. 3 (1969), 361-366.

[Ta] Y. Tashiro, Complete Riemannian manifolds and some vector fields, Trans. Amer. Math. Soc. 117 (1965), 251-275.

[Y] S.T. Yau, Isoperimetric constants and the first eigenvalue of a compact manifold, Ann. Sci. Ecole Norm. Sup. 8 (1975), 487-507.

Sebastián Montiel

Departamento de Geometría y Topología

Universidad de Granada

E-18071 Granada

Spain

e-mail: smontiel@goliat.ugr.es

(Received: October 14, 1997) 